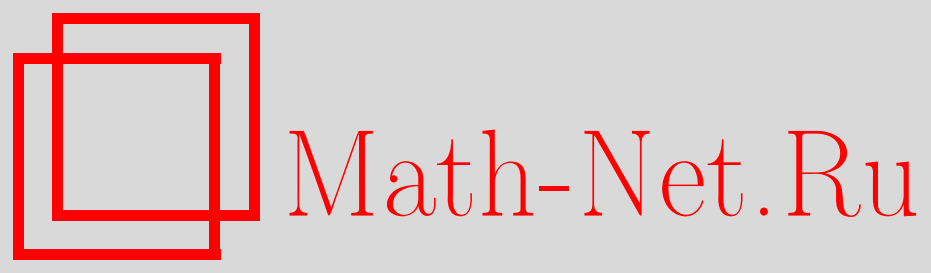

В. Клепцын, Изобретая логарифмическую линейку, Квант, 2021, номер 9, 9-12

DOI: https://doi.org/10.4213/kvant20210902

Использование Общероссийского математического портала Math-Net.Ru подразумевает, что вы прочитали и согласны с пользовательским соглашением http://www.mathnet.ru/rus/agreement

Параметры загрузки:

IP : 52.90 .164 .192

26 апреля 2023 г., 14:42:38 


\section{Изобретая логарифмическую линейку}

В.КЛЕПЦЫН

Д О КАК МИНИМУМ 1960-Х ГОДОВ логарифмическая линейка была непременной принадлежностью инженера. Она позволяла быстро выполнять различные действия - умножать, возводить в степень и многое другое, что сейчас для нас делает компьютер, калькулятор или иногда даже приложение на телефоне.

А как она была устроена? Давайте разберемся наиболее надежным способом: придумаем ее сами - а заодно научимся с ней обращаться.

Начнем с гораздо более простого вопроca: а как бы нам сделать что-нибудь, что позволяло бы одним движением складывать (небольшие) числа?

Представим себе, что мы взяли две (настоящие, деревянные!) линейки, причем на одной из них шкала у верхней границы (как это обычно бывает), а на другой у нижней (а вот так почти никогда не бывает, но можно же и самим перенести деления с одной линейки на другую). Приложим их друг к другу так, чтобы совпали нули - тогда шкалы на них совпадают (рис.1). Сдвинем теперь одну из них, нижнюю, вправо - скажем, на 2 см. Что будет тогда напротив отметки 3 см на той же линейке? Эта отметка на расстоянии в 3 см от нуля на нижней линейке - который сам находится на расстоянии в 2 см от нуля на верхней. Значит, всего эта отметка находится на расстоянии $2 \mathrm{~cm}+3 \mathrm{~cm}=5 \mathrm{~cm}$ от нуля на верхней линейке, и напротив отметки в 3 см мы видим отметку 5 см.

$\mathrm{y}_{\mathrm{pa}}$ ! Всего лишь с помощью абзаца рассуждений и двух линеек мы научились механически складывать $2+3$ и получать в ответе 5 . Но лиха беда начало!

А как складывать двузначные числа? Да точно так же. На линейке ведь обычно есть DOI: https://doi.org/10.4213/kvant20210902

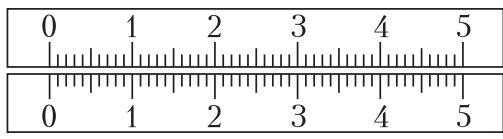
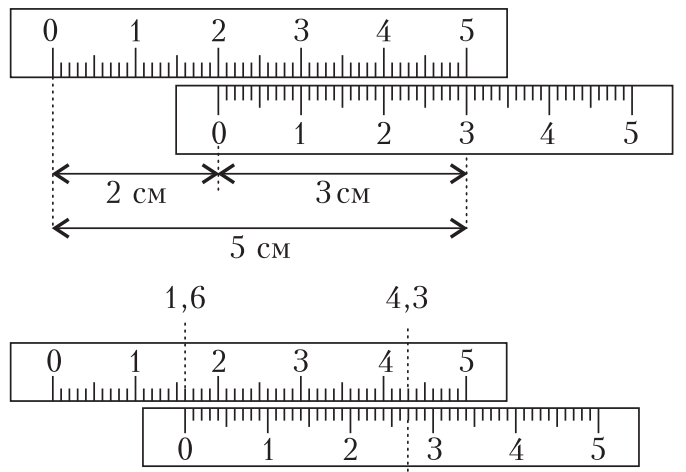

2,7

Рис. 1. Сложение с помощью двух линеек

не только сантиметровая, но и миллиметровая шкала. И можно сказать, что $16+27=$ =43, можно, что $16 \mathrm{mм}+27$ мм $=43 \mathrm{мм}$, a можно, что $1 \mathrm{~cm} 6 \mathrm{mм}+2 \mathrm{~cm} 7 \mathrm{mм}=$ $=4 \mathrm{~cm} 3 \mathrm{mм}$ (см. рис.1).

Ну хорошо. Мы научились механически, сдвигом линейки, складывать одно- и двузначные числа. Но умножать-то труднее! Как бы нам и эту операцию переложить на нашего механического помощника?

Давайте сначала представим себе, что мы хотим умножать друг на друга не любые числа, а только 10, 100, 1000 и так далее. Не то чтобы это было очень сложно: когда мы умножаем друг на друга числа вида «единица и сколько-то нулей», нам нужно просто сложить количество нулей: скажем, $100 \times 1000=100000$.

Постойте, сложить? Так это мы уже умеем! Давайте опять возьмем нашу линейку и вместо деления «1 см» поставим 10, вместо деления «2 см» поставим 100, 


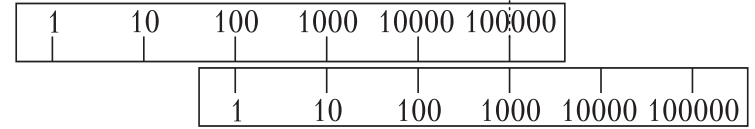

$100 \cdot 1000=100000$

Рис. 2. Умножение степеней 10

вместо деления «3 см» поставим 1000 и так далее (рис.2). И вместо нулевого деления поставим «единицу с нулем нулей», т.е. просто 1. Но вот куда нам поставить другие числа, чтобы выполнять умножение таким же сдвигом линеек?

Давайте сформулируем явно, что именно должно выполняться. Нужно, чтобы если на расстоянии $r$ см стоит число $a$, а на расстоянии $s$ см стоит число $b$, то на расстоянии $r+s$ см должно стоять число $a b$ (рис.3). А можно ли так сделать?

Наша конструкция позволяет угадать, как это надо делать. А именно: число

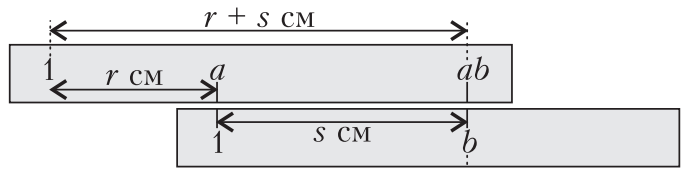

Рис. 3. Логарифмическая линейка: принцип работы

«единица с $n$ нулями» - это произведение $n$ десяток, иначе говоря, $n$-я степень 10:

$$
1 \underbrace{0 \ldots 0}_{n \text { нулей }}=\underbrace{10 \times \ldots \times 10}_{n \text { десяток }}=10^{n} .
$$

Оказывается, что можно определить и нецелую степень числа. Так, например, хотя «полраза» число само на себя умножить нельзя, но степень $\frac{1}{2}$ определена, и это просто квадратный корень из числа. И все остальные нецелые степени - хотя это дело и более сложное (и техничное) определить тоже можно. А тогда становится понятным, какой должен быть ответ: любое число $a>1$ мы хотим поставить на расстоянии в $r$ сантиметров от начала, где $r$ такое, что $10^{r}=a$. Число $r$ называют десятичным логарифмом числа $a$ - и обозначают $\log _{10} a$ (или $\left.\lg a\right)$.

Простое тождество $10^{m} \cdot 10^{n}=10^{m+n}$ выполняется и для нецелых степеней, $10^{r} \cdot 10^{s}=10^{r+s}-$ и его перевод на язык (десятичных) логарифмов дает как раз требуемое:

$$
\log _{10} a+\log _{10} b=\log _{10} a b .
$$

Теперь, чтобы сделать логарифмическую линейку, остается разметить наши две линейки, поставив каждое число $a$ на расстоянии в логарифм $a$ сантиметров (собственно, потому ее и называют - логарифмической). Или, точнее, в $\log _{10} a$, умноженном на какую-нибудь единицу длины $L$. На самом деле, единица длины 1 см слишком маленькая, и лучше взять $L$ в районе 10-15 сантиметров. На обе линейки наносится шкала, показанная на рисунке 4. Такие шкалы, что логично, тоже называют логарифмическими .

Разумеется, логарифмы бывают не только десятичные - можно зафиксировать любое основание $c$, тогда решение $x$ уравнения $c^{x}=a$ называется логарифмом $a$ по основанию с и обозначается $\log _{c} a$. Конечно же, и для них верно аналогичное (1) тождество:

$$
\log _{c} a+\log _{c} b=\log _{c} a b .
$$

Впрочем, смена основания логарифма приводит только к тому, что все результаты изменяются в одно и то же количество раз. Поэтому смена основания логарифма равносильна изменению единицы длины $L$ при построении шкалы - или, что то же самое, сжатием или растяжением шкалы в соответствующее число раз.

В настоящей логарифмической линейке обычно ее подвижная часть вставляется в своеобразный «желоб» в неподвижной, чтобы одновременно легко вдоль нее скользить и не выпадать. Самому сделать такое из дерева, конечно, не самая простая задача. Но для демонстрации принципа можно

$$
\begin{array}{llllllllllllll}
1 & 2 & 3 & 4 & 5 & 6 & 7 & 8 & 9 & 10 & 20 & 30 & 40 & 50
\end{array}
$$

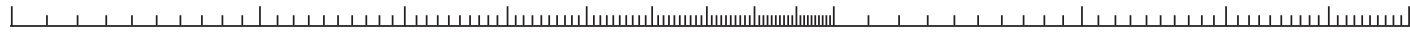




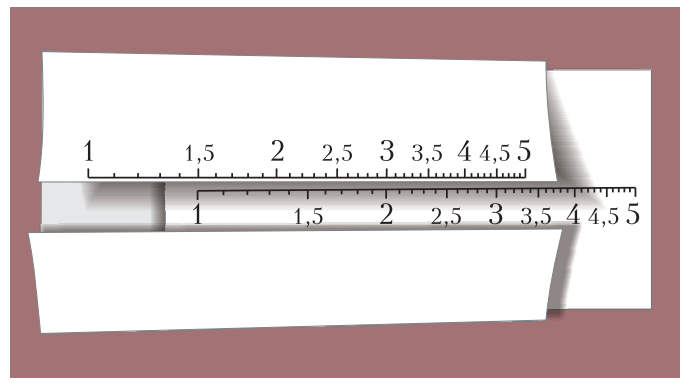

Рис. 5. Самодельная логарифмическая линейка из бумаги

сделать ее и из бумаги, «обернув» неподвижную часть вокруг подвижной (рис.5) и нанеся на каждую логарифмическую шкалу (например, воспользовавшись рисунком 4). Попробуйте это сделать! ${ }^{1}$

Как можно увидеть из фотографии на рисунке 6, настоящая логарифмическая линейка сложнее, чем наше простое описание: на ней значительно больше шкал, и благодаря этому она позволяет легко выполнять намного больше операций. Так, шкалы А (на неподвижной части) и В (на подвижной) на этой фотографии - это те самые логарифмические шкалы. Шкала D - это шкала A, сжатая в два раза. Благодаря этому можно возводить в квадрат и извлекать квадратные корни одним движением ползунка с нитью (который позволяет точно сопоставлять числа на разных шкалах). Подумайте, как именно это делается!

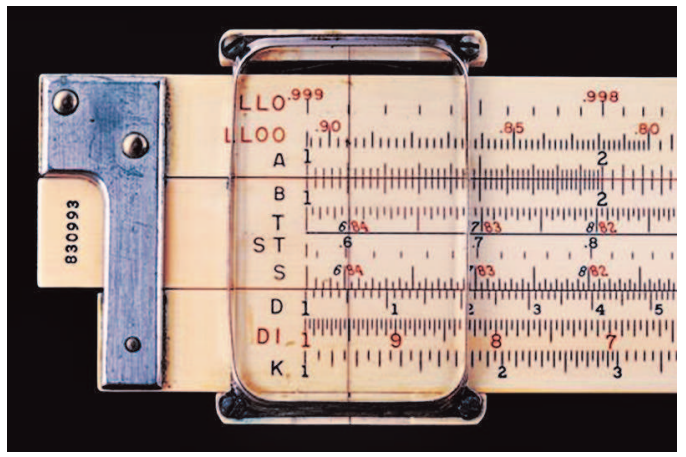

Рис. 6. Логарифмическая линейка: шкалы и нить

${ }^{1}$ Симулятор логарифмической линейки есть на сайте «Математические этюды»: см. http:// www.etudes.ru/ru/etudes/slide-rule/. Но сделать своими руками все-таки интереснее!
На некоторых шкалах (их зачастую подписывают символами LL) число $a$ стоит на расстоянии в $\log (\log a)$ единиц длины. ${ }^{2}$ Это позволяет одним движением возводить число в любую - даже нецелую! степень. Действительно, точно так же, как логарифм превращает произведение в сумму, $\log (a b)=\log a+\log b$, он превращает возведение в степень в умножение:

$$
\log \left(a^{b}\right)=b \log a \text {. }
$$

Применяя логарифм еще раз, получаем

$$
\log \log \left(a^{b}\right)=\log \log a+\log b,
$$

и поэтому для возведения в степень достаточно иметь логарифмическую шкалу и $\log \log$-шкалу, подвижные друг относительно друга (рис.7).

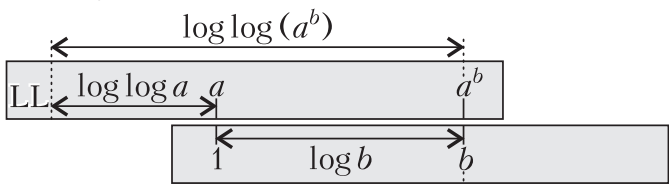

Рис. 7. Возведение в степень

Единственная тонкость - наше определение логарифма требует умения возводить в нецелую степень, а мы пока ни слова не сказали о том, как это хотя бы в принципе можно делать (не полагаясь на уже запрограммированные калькулятор или компьютер). Поэтому - давайте предъявим совсем другую, геометрическую конструкцию логарифма.

А именно, нарисуем график функции $y=\frac{1}{x}$, иными словами, гиперболу $x y=1$. И для каждого числа $a>1$ найдем площадь криволинейного четырехугольника, ограниченного осью абсцисс, нашей гиперболой и вертикальными прямыми $x=1$ и $x=a$ (рис.8). Назовем эту площадь натуральным логарифмом числа $a$ и обозначим ее через $\ln a$.

Вообще говоря, мы сейчас применили понятие «площадь» к не совсем школьному объекту: криволинейному четырехугольнику. И на уровне строгости универ-

\footnotetext{
2 Мы не уточняем основание логарифма: оно одно и то же, но для наших рассуждений неважно, каким именно мы его выберем.
} 


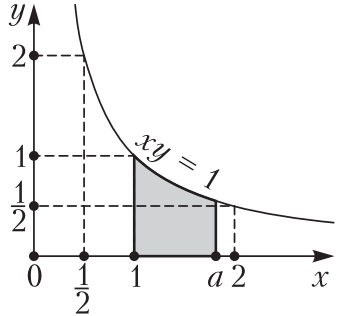

Рис. 8. Гиперболаи площадь участка ситетского курса здесь пришлось бы произносить какиенибудь страшные слова (скажем, «мера» или «интеграл»). Но с житейской точки зрения понятно, что какаято площадь у этой фигуры есть. ${ }^{3}$

Так вот - давайте каждое число $a$ поставим на расстоянии в $\ln a$ сантиметров от «начала отсчета» (в котором стоит число 1). Чтобы проверить, что при такой расстановке мы действительно сможем умножать числа простым сдвигом линейки, как показано на рисунке 3 , нам нужно проверить аналогичное (2) тождество

$$
\ln a+\ln b=\ln (a b) .
$$

И это можно доказать чисто геометрически!

Действительно, пусть $a, b>1$. Тогда $\ln (a b)$ - это площадь криволинейного четырехугольника, ограниченного осью абсцисс, гиперболой, прямой $x=1$ и прямой $x=a b$. И прямая $x=a$ разбивает эту фигуру на две части, площадь одной из которых равна $\ln a$ (эта часть на рисунке 9 слева красная). Значит, нам нужно проверить, что площадь оставшейся (синей) фигуры равна $\ln b$. Иными словами (если вспомнить, как мы определяли натуральный логарифм $b$ ), что площади двух фигур, высекаемых из области под гипербо-
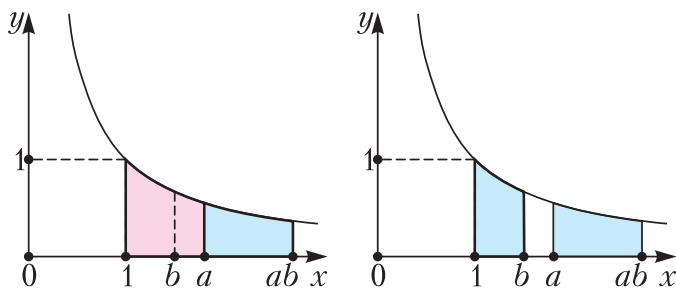

рис. 9. Проверка тождества (3)

\footnotetext{
3 Автор пользуется случаем порекомендовать читателям мультфильм «Площади фигур» на том же сайте «Математические этюды», http:// www.etudes.ru/ru/etudes/.gures-area/, посвященный как раз рассказу об определении площади не-многоугольных фигур.
}

лой неравенствами $1 \leq x \leq b$ и $a \leq x \leq a b$ (они показаны синим на рисунке 9 справа), равны.

Чтобы это проверить, давайте сожмем все по горизонтали в $a$ раз - и во столько же раз растянем по вертикали. Заметим, что вторая фигура при этом переходит в первую. Действительно, гипербола $x y=1$ переходит в себя: ведь $\left(\frac{x}{a}\right) \cdot(a y)=x y$, ось $x$ - тоже. И наконец, полоса $a \leq x \leq a b$ переходит как раз в полосу $1 \leq x \leq b$.

Но при таком преобразовании сохраняются и площади. Действительно, очень естественно ожидать, что при сжатии по горизонтали в $a$ раз площади всех фигур в $a$ раз и уменьшатся. И это действительно так. Можно в это просто поверить, а можно сказать, что это точно правда для любого прямоугольника с горизонтальными и вертикальными сторонами. А если наложить на фигуру мелкую-мелкую сетку, то можно объединением таких прямоугольников эту фигуру приблизить. Точно так же, растяжение по вертикали в $a$ раз увеличивает площади в $a$ раз. А вместе они умножают площади на $a \cdot \frac{1}{a}=1$, т.е. не изменяют.

Итак, наше преобразование сохраняет площади и переводит вторую из синих фигур на рисунке 9 справа в первую. Значит, площади этих фигур равны! Вот мы и проверили тождество (3). И тем самым научились (хотя бы теоретически!) создавать логарифмическую линейку.

Конечно же, натуральный логарифм $\ln a$ - это логарифм по некоторому основанию. Несложно понять, каким условием это основание - его обозначают буквой $e-$ задается: раз

$$
\ln e=\log _{e} e=1,
$$

то $e$ - это такая абсцисса, что площадь под гиперболой $x y=1$ от $x=1$ до $x=e$ равна 1 (рис.10).

Число $e=2,718281828459045 \ldots$ - одно из самых замечательных чисел в математике, столь же важное, как всем известное число $\pi$. 